\title{
COVID-19 Pandemic and the Verification of Effects of Yoga Intervention Using YouTube on Mental Health and Subjective Happiness of Workers
}

\author{
Juri Iwazaki, Koichiro Aoki, Chieko Kato, Keishin Kimura \\ Graduate School of Faculty of Information Science and Arts, Toyo University, Kawagoe, Japan \\ Email: cocoroshanti@yahoo.co.jp
}

How to cite this paper: Iwazaki, J., Aoki, K., Kato, C., \& Kimura, K. (2021). COVID-19 Pandemic and the Verification of Effects of Yoga Intervention Using YouTube on Mental Health and Subjective Happiness of Workers. Psychology, 12, 2083-2096. https://doi.org/10.4236/psych.2021.1212126

Received: October 21, 2021

Accepted: December 28, 2021

Published: December 31, 2021

Copyright (อ 2021 by author(s) and Scientific Research Publishing Inc. This work is licensed under the Creative Commons Attribution International License (CC BY 4.0).

http://creativecommons.org/licenses/by/4.0/ (c) (i) Open Access

\begin{abstract}
This study aimed to examine the effects on mental health and subjective well-being of busy workers during the COVID-19 pandemic, through a nine-week period of yoga practice using 30-minute videos on YouTube. A one-way analysis of variance was performed on the data obtained from the control group, the group practicing less than once a week, and the group practicing more than once a week, to examine the changes before the first week of practice and after the ninth week of practice. The results of multiple comparisons using the Tukey method showed that there was a significant difference $(p<0.05)$ between the control group and the more than once a week practice group, in the total score of GHQ12, F1 and F2 of the mood scale, and physical functioning. Significant differences $(p<0.05)$ were also found between the control group and the practice group less than once a week in F2. These results suggest that yoga practice using YouTube video may be an effective method of stress management in the Corona disaster, as a method of improving mental health and increasing positive emotions that lead to a subjective well-being.
\end{abstract}

\section{Keywords}

Yoga, Mental Health, Subjective Well-Being, Statistical Analysis, COVID-19 Pandemic, YouTube

\section{Introduction}

According to the results of a survey conducted by the Ministry of Health, Labor and Welfare in 2018, the percentage of workers who had strong anxiety, distress 
and felt stressed about their current work and professional life was as high as 58.0\% (The Ministry of Health, Labor and Welfare, 2018). The importance of supporting workers to be physically and mentally healthy has been noted (Yoneyama, Yoshida, \& Kagitani, 2019). In the 2021 edition of the World Happiness Rankings, published annually by the United Nations, it is estimated that Japan's level of happiness is $56^{\text {th }}$ out of 156 countries in the world, which is the lowest among developed countries, although it has improved from $62^{\text {nd }}$ in 2020 (United Nation, 2021). It is urgent to take measures against the stress of workers and the low sensitivity of happiness in Japan. In recent years, the number of research papers on stress management, subjective well-being, and mental health has been on the rise. Proposals on how to improve mental health and subjective well-being may be required. According to Otsuka et al., it is important for workers to be aware of their stressors and stress responses, acquire knowledge and methods for dealing with them, and implement these methods in order to promote mental health (Otsuka, Suzuki, \& Takada, 2007). Mental health is defined as a state of well-being in which individuals are able to realize their potential, cope with the normal stresses of life, work productively, and contribute to their communities (World Health Organization, 2018).

What factors are necessary for a person to be happy? It is frequently translated as subjective well-being (Terasaki, Amishima, \& Nishimura, 1999). Ed Diener, the "father of happiness studies," states that subjective well-being consists of levels of long-term pleasure, lack of discomfort, and life satisfaction (Diener, 1994). In addition, Martin Seligman, the founder of positive psychology, proposed five indicators of the elements of well-being: positive emotions, engagement, meaning and significance, positive relationships, and achievement (Seligman, 2014). It is known that happiness has two aspects: a temporary aspect as "Happy feelings" and a long-term aspect as "Level of happiness" which is a relatively long-term and stable recognition that one is happy. It has been found that the two aspects of happiness are interrelated, such that people with a high level of happiness are more likely to experience happy feelings in their daily lives, and conversely, the happier feelings they experience in their daily lives, the higher their happiness level (Institute of Physiology, 2016). This study suggests that increasing the positive emotions of workers leads to an increase in their sense of well-being, which may contribute to improving the low level of happiness among Japanese people.

Yoga is one of the approaches to maintain mental health, increase positive emotions, and improve subjective well-being. The Ministry of Health, Labor and Welfare also introduces relaxation yoga as a tip for dealing with fatigue and stress in a positive way on its mental health portal site for workers (Ministry of Health, Labor and Welfare, 2017). The population of yoga in the world is growing significantly and is currently estimated at 300 million people (Wiese, Keil, Rasmussen, \& Olesen, 2019). Arora et al. mention that the yoga helps to create a physiological state opposite to the escapist stress response, and by interrupting the stress response, a sense of balance and unity of mind and body can be 
achieved (Arora \& Bhattacharjee, 2008). Woodyard noted that one of the main goals of yoga is to acquire tranquility of mind, create happiness, relaxation, and an optimistic outlook on life, as well as to increase self-confidence, efficiency, and attentiveness, and lower irritability (Woodyard, 2011). For this reason, yoga is recommended as one of the methods for workers to obtain tranquility, reduce negative emotions, improve positive emotions, and increase their sense of well-being, away.

Bruin et al. conducted a study on workers with burnout symptoms using a combined physical exercise, restorative yoga, and mindfulness meditation (Bruin, Formsma, Frijstein, \& Bögels, 2015). The results showed significant improvements in physical and mental workability, anxiety, depression, stress, sleep quality, and positive and negative emotions after 6 weeks and 6 months, and the effect remained large and increased further over time.

Hertfiel et al. recruited 48 staff members at a British university and randomly assigned them to either a yoga group or a control group on a waiting list. The yoga group was offered 60 minutes of Dru Yoga once a week during lunchtime for six weeks, resulting in significant improvements in clarity of mind, calmness, elation, energy, and confidence compared to the control group. In addition, the yoga group reported increased life purpose and satisfaction, as well as increased confidence in stressful situations. These results indicate that even a brief program of yoga can be effective in improving emotional well-being and resilience to stress in the workplace (Hartfiel, Havenhand, Khalsa, Clarke, \& Krayer, 2010).

In a study by Murakami et al., a DVD of yoga therapy was distributed to workers for self-study at home after giving a lecture on the benefits of yoga and practicing exercises, breathing exercises, and meditation (Murakami \& Hashimoto, 2015). Comparing the physical and mental status of the 21 participants who performed the self-study and answered that yoga self-study was suitable for them before and one month after the above intervention, there was a significant improvement in irritability and physical complaints, and a marginally significant improvement in fatigue. Therefore, stress management intervention through yoga therapy could be effective as a stress management method adding some modifications such as the promotion of self-study.

Sakuma et al. randomly allocated 19 working women including nurses, caregivers, and clerical staff to a yoga group $(\mathrm{n}=12)$ or a control group $(\mathrm{n}=7)$ and the effects of yoga were examined (Sakuma, Miyauchi, Sasaki, Usui, \& Sato, 2014). The participants in the yoga group were given an 8-week program with a 20 -minute viewing of a yoga DVD while physical function, physical and mental discomfort, and symptoms associated with the menstrual cycle were measured. As a result, significant differences were found in "swelling of hands and feet", "back-ache" and "irritation," which indicated that yoga was considered to be effective in recovering from fatigue. Yoga can be an effective method of managing the body and mind, but there is still little scientific evidence on short programs that are easy to engage in. In the past, yoga classes have been given to workers, and although they have felt the effects, few workers have been able to continue 
self-practicing afterwards.

Above all, the world is changing at a dizzying speed since the COVID-19 pandemic was announced (Figueiredo, Sandre, Portugal, Oliveira, Chagas, Raony et al., 2020). In a survey conducted by the International Committee of the Red Cross (ICRC) in September 2020, more than half (51\%) of respondents said that the spread of COVID-19 has had a negative impact on their mental health (ICRC, 2020). It also argued that the COVID-19 pandemic is clearly associated with significant mental stress and that reducing its detrimental effects on mental health is an international public health priority (Xiong, Lipsitz., Nasri, Lui., Gill, \& Phan, 2020). In Japan, at a regular press conference of the Tokyo Metropolitan Medical Association in October 2020, it was reported that the number of suicides in Japan in August, compared to the average for the same month in 2017-19, was 356 for men under 40, a 31.4\% increase over the previous year, and 189 for women, a significant increase of $76.6 \%$ over the previous year (Nihon Keizai Shimbun, 2020).

COVID-19 has also various psychological effects on health care workers. A survey of 2638 HCWs from all hospitals in the West Midlands, UK, between June 5,2020 , and July 31,2020 , revealed symptoms of anxiety (34.3\%), depression (31.2\%), and PTSD (24.5\%). After the initial peak of the COVID-19 pandemic in the UK, it was reported that the rate of clinically significant mental health symptoms was higher among hospital HCWs, and furthermore, those with a history of mental illness were at highest risk (Wanigasooriya, Palimar, Naumann, Ismail, Fellows, Logan, Thompson, Bermingham, Beggs, \& Ismail, 2021). Reger et al. also contend that it is important to consider the long-term mental health implications for HCWs in this crisis (Reger, Piccirillo, \& Buchman-Schmitt, 2020). This is because medical personnel are on the frontlines-risking their welfare while society at large undertakes unprecedented social distancing. Even in this emergency circumstance, or especially in this emergency circumstance, we neglect mental health at our peril and to our long-term detriment (Cullen, Gulati, \& Kelly, 2020).

The Centers for Disease Control and Prevention (CDC) suggests deep breathing, stretching, meditation, and regular exercise as healthy ways to cope with stress, also mentions the importance of time to free the mind (Centers for Disease Control \& Prevention, 2019).

Nagendra suggests that all yoga practices utilize deep relaxation which could release all tensions and stresses to prevent immune suppression that would otherwise have weakened the responses to the onslaught of infectious bacteria and viruses (Nagendra, 2020). However, Ramdas argues that no studies were found exploring the potential role of Yoga as an intervention for mental disorders during COVID-19 pandemic (Ramdas, 2020). We need to practice an antidote for stress-at work or even at home-at the time of experiencing the stress, not 5 hours later when we can cloister ourselves in a room for a short time.

Sahni et al. investigated the effect of yoga practice on illness perception, and the wellbeing of healthy adults during $4-10$ weeks of lockdown due to a 
COVID-19 outbreak in India (Sahni, Singh, Sharma, \& Garg, 2021). The 668 participants were grouped as yoga practitioners, other spiritual practitioners, and non-practitioners, based on their responses to daily practices that they follow. Multivariate analysis indicates that yoga practitioners had significantly lower depression, anxiety, \& stress (DASS), and higher general wellbeing (SWGB) as well as higher peace of mind (POMS) than the other two groups. The results further revealed that yoga practitioners significantly differed in their perception of personal control, illness concern and the emotional impact of COVID-19. Evidence supports that yoga was found to be an effective self-management strategy to cope with stress, anxiety and depression, and to maintain wellbeing during a COVID-19 lockdown.

Considering these facts, the present study was undertaken in order to clarify the effects of simple 9-week yoga programs using YouTube on physical functioning and psychological state, mental health, and the subjective well-being of workers in the COVID-19 pandemic, which makes face-to-face instruction difficult.

\section{Preliminary Survey}

A preliminary survey was conducted on 22 practitioners at Yoga Studio A. A method of a main survey is considered based on the results of this survey. The purpose was to examine the appropriateness of the YouTube video and Word file still image training methods, the structure of the program, and the survey items.

\subsection{Method}

In July 2020, the participants practiced a yoga program for about 20 minutes once a week for 4 weeks. Group A $(n=15)$ received a YouTube URL of the yoga program video once a week. Group B $(n=7)$ received a Word file with still images of the same program. The date and number of practice sessions were optional for the participants and were reported to the researcher. Participants were asked to enter their physical functions, changes in psychological state comparing before and after, and positive and negative points of their practice in a weekly questionnaire on Google Form. The questionnaire consisted of five options: 1) not at all, 2) no, 3) a little, 4) a lot, and 5) severe. The participants were asked to select the changes in physical functioning (stiff shoulders, back pain, coldness, lethargy, headache, fatigue) and psychological state (tension, anxiety, irritability, depression, anger, confusion). They were also asked to select from 1 to 10 for the question "How happy are you?"

\subsection{Results}

The values of physical function (stiff shoulders, back pain, coldness, sluggishness, headache, fatigue), psychological state (tension, anxiety, irritability, depression, anger, confusion), and happiness entered by the participants before 
and after each yoga practice were combined in Group A $(\mathrm{n}=15)$ and Group B ( = 7) for Group A (15 participants) and Group B (7 participants), and the mean values were calculated (see Table 1). Both teams found that there were positive changes in physical functioning, psychological state, and happiness after the yoga practice. Stiff shoulders: Group A $3.10 \rightarrow 2.56(0.54)$, Group B $2.79 \rightarrow 2.29$ $(-0.5)$. Back pain: Group A $2.79 \rightarrow 2.18(-0.61)$, Group B $2.50 \rightarrow 2.18(-0.32)$. Coldness: Group A $2.51 \rightarrow 1.84(-0.67)$, Group B $2.61 \rightarrow 2.18$ (-0.43). Lethargy: Group A $2.80 \rightarrow 2.08(-0.72)$, Group B $2.96 \rightarrow 2.11(-0.85)$. Headache: group A $2.13 \rightarrow 1.82(-0.31)$, Group B $2.11 \rightarrow 1.79(-0.32)$. Fatigue: group A $2.82 \rightarrow 2.07$ $(-0.75)$, Group B $3.00 \rightarrow 2.29(-0.71)$. Tension: Group A $2.30 \rightarrow 1.74(-0.56)$, Group B $2.64 \rightarrow 2.07(-0.57)$. Anxiety: Group A $2.61 \rightarrow 2.00(-0.61)$, Group B $2.96 \rightarrow 2.43$ (-0.53). Irritability: Group A $2.56 \rightarrow 1.79(-0.77)$, Group B $2.68 \rightarrow$ 1.96 (-0.72). Depression: Group A $2.31 \rightarrow 21.79(-0.77)$, Group B $2.39 \rightarrow 1.96$ $(-0.43)$. Anger: Group A $2.25 \rightarrow 1.72(-0.53)$, Group B $2.11 \rightarrow 1.71(-0.4)$. Confusion: Group A $2.28 \rightarrow 1.96(-0.48)$, Group B $2.36 \rightarrow 1.96(-0.4)$. Happiness: Group A $7.33 \rightarrow 8(+0.67)$, Group B $7 \rightarrow 7.29(+0.29)$.

\subsection{Discussions}

These results suggest that physical function, psychological function, and sense of happiness may be improved in both groups after yoga practice. However, according

Table 1. The average of the values before and after the yoga practice of group A $(n=15)$ and group $B(n=7)$ combined for 4 weeks.

\begin{tabular}{ccccc}
\hline & \multicolumn{2}{c}{ Gr. A } & \multicolumn{2}{c}{ Gr. B } \\
\cline { 2 - 5 } & before & after & before & after \\
\hline Physical functioning & & & & \\
\hline Stiff shoulders & 3.10 & 2.56 & 2.79 & 2.29 \\
Back pain & 2.79 & 2.18 & 2.50 & 2.18 \\
Coldness & 2.51 & 1.84 & 2.61 & 2.18 \\
Lethargy & 2.80 & 2.08 & 2.96 & 2.11 \\
Headache & 2.13 & 1.82 & 2.11 & 1.79 \\
Fatigue & 2.82 & 2.07 & 3.00 & 2.29 \\
\hline Psychological state & & & & \\
\hline Tension & 2.30 & 1.74 & 2.64 & 2.07 \\
Anxiety & 2.61 & 2.00 & 2.96 & 2.43 \\
Irritability & 2.56 & 1.79 & 2.68 & 1.96 \\
Depression & 2.31 & 1.79 & 2.39 & 1.96 \\
Anger & 2.25 & 1.72 & 2.11 & 1.71 \\
Confusion & 2.28 & 1.80 & 2.36 & 1.96 \\
Happiness & 8.00 & 7.00 & 7.29 \\
\hline & & & & \\
\hline & & & & \\
\hline
\end{tabular}


to the descriptions of participants, about 20 minutes of practice was too short, and a longer practice was needed. The Word practice was complicated and difficult for beginners. Therefore, this survey will be conducted using only the practical training method of about 30 minutes and using YouTube videos.

\section{Main Survey}

The survey covered public interest workers (men and women) such as local government officials and health care workers (HCW). In the first week, participants answered a questionnaire and a survey using Google Form. After that, a one-hour explanation of the study followed by a yoga practice is given online using Zoom or on YouTube. This will be followed by a 30-minute YouTube yoga video sent once a week for 8 weeks. The number of practice sessions will be at the discretion of the participant. After the ninth week of practice, participants will complete a Google Form and questionnaire to survey physical functioning, psychological state, frequency of practice, positive and negative points of the practice, and any changes in life events. Differences in the frequency of practice will be investigated. Although this design is based on the pre-liminary survey, it may be changed.

Based on the results of the preliminary investigation, which suggested that the video-based yoga practice improved physical functioning, psychological functioning, and subjective well-being. The purpose of this study was to examine further examine the program and verify the effects of yoga on mental health and subjective well-being using YouTube.

\subsection{Method}

The survey period was from November 18, 2020 to May 2, 2021. The research participants were male and female workers with high public interest such as local civil servants and health care workers. There were 82 participants in the study. The survey was conducted on 60 participants who responded to the questionnaire in the $9^{\text {th }}$ week. 15 of the 60 participants were in the control group, who were not able to participate in yoga training but wished to participate in the research. The first week, the participants answered a questionnaire and a Google form using their nicknames. Afterwards, a one-hour explanation of this study and yoga and yoga practice was given online using Zoom or on YouTube. One 30-minute YouTube yoga video per week was transmitted for 8 weeks of practice. The number of practice sessions was up to the participants. After the ninth week of practice, participants were asked to fill out a Google form and questionnaire to survey their physical functioning, psychological state, number of practices, positive and negative aspects of the practice, and any changes in life. Differences in the frequency of practice were investigated.

Kimura claims that clients need yoga for stress release, relaxation (liberation of the mind), expansion of awareness, multifaceted and flexible thinking (selection of alternatives), and behavioral change (transformation of the meaning of life) (Kimura, 2020). The content of this study consists of intellectual education, 
gymnastics, breathing exercises, and Vedic meditation. Intellectual education refers to education that leads to ideal psychological effects. Vedic meditation is a four-stage meditation technique that has been handed down in India from the Upanishads to the present day, which is 1 . Listen to the meditational detail, 2. Consider it by yourself, 3. Deep meditational state in everyday life, 4 . Awareness (Kimura, 2017).

\subsubsection{Research Items}

In this survey, the following scores (i) to (vii) were calculated.

1) Questionnaire items regarding physical and mental conditions

(i) Physical function: stiff shoulders, backache, coldness, lethargy, headache, and fatigue, with a 6-point scale from 0 (not at all) to 5 (very severe).

(ii) Psychological state: Nervousness, anxiety, irritability, depression, anger, and confusion were assessed using a 6-point scale from 0 (not at all) to 5 (very severe).

(iii) Happiness: How happy are you now? Participants scored their happiness on a scale of (1) to (10).

\section{2) 12-Item General Health Questionnaire (GHQ-12)}

GHQ-12 is a screening test which is useful for identifying, evaluating, and detecting symptoms of neurological patients. This questionnaire consists of question items asking if there have been mental and physical problems related to one's health status from two or three weeks ago to the present. This questionnaire is easy for both individuals and groups to answer in a short time in hospitals, schools, companies, etc.

3) Mood scale (Fukuoka, 2011)

The mood state scale consists of 20 items (F1:10 positive and F2: 10 negative items each), which were created by Fukuoka et al. based on the POMS with some additions and modifications and is answered on a 3-point scale (1. not at all, 2. a little, 3. exactly) (Fukuoka, 2011).

4) The Japanese version of the Subjective Happiness Scale (SHS: Shimai Ohtake, Utsugi, \& Ikemi, 2004)

SHS is a scale for the purpose of measurement of subjective happiness with relatively few questions constituting a single-factor. The score of SHS can be obtained by calculating an average score of seven-point scale (Shimai, Ohtake, Utsugi, \& Ikemi, 2004). However, considering that busy workers can easily answer the questionnaire, it was decided to use the "subjective happiness" scale for adolescents (Sogabe \& Motomura, 2010). It is based on Shimai et al.'s scale, and after preliminary investigation, the wording was improved to make it clearer in Japanese, and obtain responses using a four-point scale: I strongly agree (4 points), I agree (3 points), not so much (2 points), and do not agree at all (1 point).

\section{5) Week 1 questionnaire survey items}

Participants in the 1st week will be asked to fill out a Google Form questionnaire before yoga practice. The survey items are as follows: (1) Nickname, (2) 
Email address, (3) Date of practice, (4) Medications you are taking or have been taking, (5) What are you looking for in yoga? (6) Physical functioning (stiff shoulders, back pain, coldness, lethargy, headache, fatigue), psychological state (tension, anxiety, irritability, depression, anger, confusion) from (0. none to 5. severe), (7) How happy are you? (Choose from 1 to 10).

\section{6) Week 9 questionnaire survey items}

Immediately after the yoga practice in the 9th week, participants will fill out a Google Form survey. The survey items are as follows: (1) Nickname (2) Email address (3) Age and gender (4) If you have practiced yoga, the date and time of practice (5) Frequency of yoga practice: Choose from the first week to the ninth week (did not practice, practiced once, practiced two or more times) (6) Reason for not practicing (busy, no time, boring, not necessary, difficult, had problems after practicing, other, etc.) (7) Positive and negative points of the practice. (free answer) (8) Enter the physical function and psychological state immediately after the practicing yoga. The content of the questionnaire is physical functioning (stiff shoulders, back pain, coldness, lethargy, headache, fatigue) and psychological state (tension, anxiety, irritability, depression, anger, confusion), which can be selected from (0. none to 5. severe) (9) How was the yoga practice? (very good, good, average, not good, not good at all) (10) How happy are you? (Choose from 1 to 10) (11) Have you experienced any significant changes in your life? (None, change in responsibility at work, change in working conditions, trouble with boss, marriage, divorce, pregnancy, childbirth, accident, moving, death of a close person, separation from a lover, illness or serious injury, big change in family health, big change in finances, the son or daughter left home, and others).

\subsubsection{Analysis Method}

The participants were divided into three groups: the control group (15 participants), the group that practiced less than once a week (less than once a week practice group: 19 participants), and the group that practiced more than once a week (more than once a week practice group: 26 participants). For the scores (1) to (7) obtained by the questionnaire survey, the difference between the scores before the first week of practice and after the ninth week of practice for each subject were calculated. A one-way ANOVA was conducted on the pre/post differences in these scores, with the above three groups as factors. IBM SPSS Statics 26 was used for the analysis, and the level of significance was set at less than 0.05.

\subsubsection{Ethical Considerations}

This study was approved by the Ethics Committee of the Japan Yoga Therapy Society, and participants were surveyed after the outline of the study was explained and consent was obtained.

\section{Results}

Of the 45 valid respondents, 19 (42\%) practiced yoga less than once a week and 
$26(58 \%)$ practiced yoga more than once a week. The control group with no yoga practice consisted of 15 respondents (see Table 2).

A one-way analysis of variance was performed on the differences in scores (i) through (vii) before and after the experiment, with the control group, the practice less than once a week group, and the practice more than once a week group as factors. $2(F(2,47)=3.998, p<0.05)$, and the total score for physical condition $(F(2,47)=5.188, p<0.01)$.

The results of multiple comparisons using the Tukey method showed that there was a significant difference $(p<0.05)$ between the control group and the more than once a week practice group, in the total score of GHQ12, and the score decreased significantly in the more than once a week practice group. In the

Table 2. Changes in scores before and after the experiment in each group (1. Control group, 2. Less than once a week practice group, 3. More than once a week practice group).

\begin{tabular}{|c|c|c|c|c|c|}
\hline Score & Group & $\mathbf{M}$ & SD & $\begin{array}{c}\text { F-value } \\
(2,47)\end{array}$ & $\begin{array}{l}\text { Results of Multiple } \\
\text { Comparison }\end{array}$ \\
\hline \multirow{3}{*}{$\begin{array}{c}\text { GHQ12 } \\
\text { Total difference }\end{array}$} & 1 & -1.8 & 7.38 & \multirow{3}{*}{$8.249^{* *}$} & \multirow{3}{*}{$1<2,3$} \\
\hline & 2 & 3.86 & 4.33 & & \\
\hline & 3 & 6.96 & 6.87 & & \\
\hline \multirow{3}{*}{$\begin{array}{l}\text { Mood Scale F1 } \\
\text { Total difference }\end{array}$} & 1 & -1.4 & 5.5 & \multirow{3}{*}{$6.13^{\star *}$} & \multirow{3}{*}{$1<3$} \\
\hline & 2 & 2.71 & 4.46 & & \\
\hline & 3 & 4.81 & 5.24 & & \\
\hline \multirow{3}{*}{$\begin{array}{l}\text { Mood Scale F2 } \\
\text { Total difference }\end{array}$} & 1 & -2.4 & 5.64 & \multirow{3}{*}{$4.05^{\star}$} & \multirow{3}{*}{$1<2,3$} \\
\hline & 2 & 3.36 & 5.03 & & \\
\hline & 3 & 2.92 & 5.67 & & \\
\hline \multirow{3}{*}{$\begin{array}{c}\text { SHS } \\
\text { Total difference }\end{array}$} & 1 & -0.6 & 2.21 & \multirow{3}{*}{2.315} & \multirow{3}{*}{$1,2<3$} \\
\hline & 2 & 0.57 & 1.6 & & \\
\hline & 3 & 0.77 & 1.21 & & \\
\hline \multirow{3}{*}{$\begin{array}{l}\text { Physical function } \\
\text { Total difference }\end{array}$} & 1 & -0.7 & 8.26 & \multirow{3}{*}{$5.188^{\star *}$} & \multirow{3}{*}{$1,2<3$} \\
\hline & 2 & -3.43 & 7.08 & & \\
\hline & 3 & -8.58 & 6.83 & & \\
\hline \multirow{3}{*}{$\begin{array}{l}\text { Psychological state } \\
\text { Total difference }\end{array}$} & 1 & -0.3 & 9.02 & \multirow{3}{*}{1.701} & \\
\hline & 2 & -4.14 & 7.17 & & \\
\hline & 3 & -5.5 & 7.21 & & \\
\hline \multirow{3}{*}{$\begin{array}{c}\text { Happiness } \\
\text { Total difference }\end{array}$} & 1 & -0.1 & 2.38 & \multirow{3}{*}{1.411} & \\
\hline & 2 & 1.14 & 1.96 & & \\
\hline & 3 & 1.08 & 1.92 & & \\
\hline
\end{tabular}

${ }^{\star} p<0.05,{ }^{* *} p<0.01$. 
mood scale, there was a significant difference $(p<0.05)$ between the control group and the more than once a week practice group in the total score of F1, and the score increased significantly in the more than once a week practice group. There was a significant difference in the total F2 scores between the control group and the less than once a week practice group and the more than once a week practice group $(p<0.05)$, with a greater decrease in scores in the practice group. In the total score of physical function, there was a significant difference $(p<0.05)$ between the control group and the more than once a week practice group, and the score decreased greatly in the more than once a week practice group.

\section{Discussions}

In this study, we provided yoga practice in video format for approximately 30 minutes for 9 weeks, at a time when face-to-face yoga instruction is difficult and for busy workers to practice anytime and anywhere. The results showed that the yoga practice improved mental health, increased positive emotions, decreased negative emotions, and improved physical functioning.

These results are in line with those of Bruin et al. who showed significant improvements in positive and negative emotions of workers in a program that included yoga and mindfulness (Reger, Piccirillo, \& Buchman-Schmitt, 2020). The results were comparable to the studies conducted by Murakami et al. and Sakuma et al. on workers using yoga videos, which showed improvements in physical complaints and negative emotions (Murakami \& Hashimoto, 2015, Sakuma, Miyauchi, Sasaki, Usui, \& Sato, 2014). In addition, our study showed the effect of improving positive emotions that lead to a sense of well-being, which has not been clarified in previous studies by them. In addition, our study showed the effect of improving positive emotions that lead to a sense of well-being, which has not been clarified in previous studies by them. In the COVID-19 pandemic, our study found comparable efficacy to a study by Sahni et al. during the lockdown in India, which supported that yoga was found to be an effective self-management strategy for coping with stress, anxiety and depression and maintaining wellbeing (Sahni, Singh, Sharma, \& Garg, 2021).

One of the reasons for this result is that many of the local government officials and healthcare workers had entered the Corona disaster period and were mentally exhausted due to various changes in their lives and work. Many of them continued the yoga practice in the video relatively earnestly despite this period. This could be due to the relative appropriateness of the program, the fact that yoga is not just exercise or stretching, or the fact that the stress management methods of the Corona disaster were needed. The reasons why some participants were not able to continue the program may be that their mental state was relatively healthy at the beginning of the program and they did not feel the need for it, the duration of the program ( 9 weeks), the time required (30 minutes), and the difficulty in establishing a trusting relationship with the instructor compared 
to face-to-face yoga.

A significant trend in subjective well-being was observed between the control group and the once-a-week or more group, which may be clarified by increasing the number of participants or conducting a long-term survey. During the period of participation in this study, some participants experienced major changes in their lives, such as change in work responsibility, major change in the health of a family member, problem with their supervisors, so busy and came to work almost every day, even on the days off, working conditions changed, major economic change, Transfer to the corona ward, and Death of someone close relative. Although they were in the midst of these changes, some of them described positive mental effects such as feeling mentally stable, feeling at ease, and feeling refreshed by continuing the yoga practice. This may have been a means of changing their minds by taking a break from stressful situations and confronting themselves.

The free descriptions of the positive and negative aspects of yoga practice were categorized and tabulated as follows. Positive comments (number of people): mental (18), physical (16), breathing (2), meditation (0). Negative comments (number of participants): time (7), problems specific to video (3), body (2), mind (2), and meditation (0). None of the participants commented on the meditation. It may be that it was difficult for beginners to reach the meditation stage in the video practice as opposed to face-to-face practice. In future research, it may be better to introduce the meditation gradually. These results suggest that yoga practice using YouTube video may be an effective method of stress management in the Corona disaster, as a method of improving mental health and increasing positive emotions that lead to a subjective well-being.

\section{Conclusion}

The study shows that practicing yoga for nine weeks with a 30-minute video on YouTube can improve the mental health of workers, including HCW and local government officials, and increase positive emotions that lead to subjective well-being, despite being in the middle of the COVID-19 pandemic, which calls for social distancing and refraining from going out.

\section{Conflicts of Interest}

The authors declare no conflicts of interest regarding the publication of this paper.

\section{References}

Arora, S., \& Bhattacharjee, J. (2008). Modulation of Immune Responses in Stress by Yoga. International Journal of Yoga, 1, 45-55. https://doi.org/10.4103/0973-6131.43541

Bruin, I. E., Formsma, R. A., Frijstein, G., \& Bögels, M. S. (2017). Mindful2Work: Effects of Combined Physical Exercise, Yoga, and Mindfulness Meditations for Stress Relieve in Employees. A Proof of Concept Study. Mindfulness, 8, 204-217.

https://doi.org/10.1007/s12671-016-0593-x 
Centers for Disease Control \& Prevention (2019). Coping with Stress Pandemics Can Be Stressful.

https://www.cdc.gov/coronavirus/2019-ncov/daily-life-coping/managing-stress-anxiety . html

Cullen, W., Gulati, G., \& Kelly, B. D. (2020). Mental Health in the COVID-19 Pandemic. QJM: An International Journal of Medicine, 113, 311-312.

https://doi.org/10.1093/qjmed/hcaa110

Diener, E. (1994). Assessing Subjective Well-Being: Progress and Opportunities. Social Indicators Research, 31, 103-157. https://doi.org/10.1007/BF01207052

Figueiredo, C., Sandre, P., Portugal, L., Oliveira, T., Chagas, L., Raony, I., Ferreira, E., Araujo, E., Santos, A., \& Bomfima, P. (2020). COVID-19 Pandemic Impact on Children and Adolescents' Mental Health: Biological, Environmental, and Social Factors. Progress in Neuro-Psychopharmacology and Biological Psychiatry, 106, Article ID: 110171. https://doi.org/10.1016/j.pnpbp.2020.110171

Fukuoka, K. (2011). Relationship between Interpersonal Interaction and Mood State Associated with Daily Stress Experiences: A Preliminary Examination of the Mood Scale. Kawasaki Journal of Health and Welfare, 20, 475-479.

Hartfiel, N., Havenhand, J., Khalsa, S., Clarke, G., \& Krayer, A. (2011) The Effectiveness of Yoga for the Improvement of Well-Being and Resilience to Stress in the Workplace. Scandinavian Journal of Work, Environment \& Health, 37, 70-76.

https://doi.org/10.5271/sjweh.2916

Institute of Physiology (2016). Relationship between Happiness and the Brain Revealed-Hopes for Improving the Happiness of the Japanese People.

https://www.nips.ac.jp/release/2016/07/post 321.html

Kimura, K. (2017). Yoga Therapy Theory. Japan Yoga Niketan.

Kimura, K. (2020). Five Thousand Years of Psychotherapy in India-Yoga Therapy Darshanar. Gaia Books.

Ministry of Health, Labour and Welfare (2017). Ear for Mind: Mental Health Portal Site for Working People. https://kokoro.mhlw.go.jp/ps/relaxation/

Murakami, S., \& Hashimoto, S. (2015). Stress Management Interventions with Yoga Therapy. Japan Society of Health and Behavioral Sciences, 29, 42-49.

Nagendra, H. R. (2020). Yoga for COVID-19. International Journal of Yoga, 13, 87-88. https://doi.org/10.4103/ijoy.IJOY $27 \quad 20$

Nihon Keizai Shimbun (2020). Women's Suicides Rise, Care Work Weighs on Corona. https://www.nikkei.com/article/DGXMZO66453340Q0A121C2TY5000

Otsuka, Y., Suzuki, A., \& Takada, M. (2007). Recent Trends in Workplace Mental Health and the Practice of Workplace Stress Measures Focusing on Stress Coping. The Japanese Journal of Labor Research, 49, 41-53.

Ramdas, R. et al. (2020). Yoga for COVID-19 and Natural Disaster Related Mental Health Issue: Challenges and Perspectives. Asian Journal Psychiatry, 53, Article ID: 102386. https://doi.org/10.1016/j.ajp.2020.102386

Reger, M. A., Piccirillo, M. L., \& Buchman-Schmitt, J. M. (2020). COVID-19, Mental Health, and Suicide Risk among Health Care Workers: Looking beyond the Crisis. The Journal of Clinical Psychiatry, 81, Article ID: 20com13381.

Sahni, P.S., Singh, K., Sharma, N., \& Garg, R. (2021). Yoga an Effective Strategy for Self-Management of Stress-Related Problems and Wellbeing during COVID19 Lockdown: A Cross-Sectional Study. PLoS ONE, 16, e0245214.

https://doi.org/10.1371/journal.pone.0245214 
Sakuma, Y., Miyauchi, S., Sasaki, M., Usui, A., \& Sato, C. (2014). Effectiveness of a Simple Yoga Program in Women Workers. Journal of the Japanese Society of Health Medicine, 23, 69-79.

Seligman, E. P. M. (2014). Positive Psychology's Challenge: From 'Happiness' to Lasting Happiness Tokyo, Discover Twenty-One (pp. 33-37).

Shimai, T., Ohtake, E., Utsugi, S., \& Ikemi, Y. (2004). An Examination of the Re-liability and Validity of the Japanese Version of the Subjective Happiness Scale (SHS). Journal of the Japan Society of Public Health, 51, 845-853.

Sogabe, K., \& Motomura, M. (2010). Subjective-Happiness-Feeling of University Student in Adolescent Stage toward an Exploration of Its Influential Factors. Wakayama University Faculty of Education Minutes, Educational Sciences, 60, 81-87.

Terasaki, S., Amishima, K., \& Nishimura, T. (1999). The Structure of Subjective Happiness. Kawasaki Journal of Health and Welfare, 9, 43-48.

The Ministry of Health, Labor and Welfare (2018). Status of Working Hours and Mental Health Measures, etc.

https://www.mhlw.go.jp/wp/hakusyo/karoushi/20/dl/20-1-2.pdf

United Nation (2021). The World Happiness Report 2021. https://worldhappiness.report/ed/2021/happiness-trust-and-deaths-under-COVID-19/ ?fbclid=IwAR04odcLpAAphtXjpzdz8cCQK9OUlegF9hciX- hmsQz8vbvdJXYC6mus1g

Wanigasooriya, K., Palimar, P., Naumann, N. D., Ismail, K., Fellows, L. J., Logan, P., Thompson, V. C., Bermingham, H., Beggs, D. A., \& Ismail, T. (2021). Mental Health Symptoms in a Cohort of Hospital Healthcare Workers Following the First Peak of the COVID-19 Pandemic in the UK. Cambridge University Press Public Health Emergency Collection. https://doi.org/10.1192/bjo.2020.150

Wiese, C., Keil, D., Rasmussen, A. S., \& Olesen, R. (2019). Effects of Yoga Asana Practice Approach on Types of Benefits Experienced. International Journal of Yoga, 12, 218-225. https://doi.org/10.4103/ijoy.IJOY $81 \quad 18$

Woodyard, C. (2011). Exploring the Therapeutic Effects of Yoga and Its Ability to Increase Quality of Life. International Journal of Yoga, 4, 49-54. https://doi.org/10.4103/0973-6131.85485

World Health Organization (2018). Mental Hearth: Strengthening Our Response. https://www.who.int/news-room/fact-sheets/detail/mental-health-strengthening-our-r esponse

Xiong, J., Lipsitz, O., Nasri, F., Lui, L. M.W., Gill, H., Phan, L., Chen-Li, D., Iacobucci, M., Ho, R., Majeed, A., \& McIntyre, S. R. (2020). Impact of COVID-19 Pandemic on Mental Health in the General Population: A Systematic Review. Journal of Affective Disorders, 277, 55-64. https://doi.org/10.1016/j.jad.2020.08.001

Yoneyama, M., Yoshida, H., \& Kagitani, Y. (2019). The Relationship between Occupational Stress and Perceptions of the Organization. Science of Mind-Body Health, 15, 71-81. 